\title{
Producir y consumir: la logística, clave del éxito de una cadena de moda
}

\author{
Production and consumption: \\ the logistic, key to success of a fashion chain
}

\author{
Itziar Agulló Fernández \\ Grupo de investigación Charles Babbage \\ en Ciencias Sociales del Trabajo \\ tziagullo@yahoo.es
}

Recibido: 16.06.2011

Aprobado definitivamente: 13.12.2011

\begin{abstract}
RESUMEN
¿Cómo una empresa puede llegar a alcanzar la importancia de Zara en un lapso de tiempo tan breve? Entre otro de los elementos que han hecho posible el éxito indiscutible de Zara, aparece el papel preponderante de la logística, no sólo como transporte y almacenaje sino también -y fundamentalmente- como un complejo entramado de información fluida entre las partes que la componen: el diseño, la fabricación de las prendas y las tiendas en que éstas se venden. Desde la perspectiva pro-empresarial encontramos un elevado número de estudios que se han venido haciendo a lo largo de los últimos años con respecto al modelo del Grupo Inditex -del que Zara es el caso más representativo - y que ha querido ser un modelo de negocio del que 'toda empresa tiene algo que aprender'. Aquellos estudios, entre otras cosas, desmenuzan el funcionamiento de la logística haciendo patente que es la clave del éxito de esta gran cadena de moda. En cambio, en esas explicaciones, los trabajadores quedan invisibilizados. ¿Qué condiciones de trabajo han de soportar los empleados de la logística? ¿Existe una intensificación del trabajo a causa de los ritmos que se imponen? ¿Qué papel juegan los sindicatos? Mediante esta investigación se ha tratado de dar respuesta a estas y otras preguntas.
\end{abstract}

Palabras Clave: Logística, información fluida, flexibilización, condiciones de trabajo, justo a tiempo, Zara.

\footnotetext{
ABSTRACT

How a company can reach the importance of Zara in a very short period of time? Among other elements that have made possible the massive success of Zara, you see the role of logistics, not only as transportation and storage but also, and fundamentally, as a complex network of information flow between the parties

${ }^{1}$ Este artículo se basa en una parte de la Tesis doctoral "La precariedad en los mercados de trabajo y consumo de los jóvenes. El caso Zara", que la autora defendió el 26 de noviembre de 2010.

La autora agradece las sugerencias realizadas por parte de los evaluadores de la revista para la redacción final de este artículo.
} 
that compose it: design, manufacture of clothing and stores in which they are sold. Since the pro-business perspective are a large number of studies have been done over recent years with respect to the model of the Inditex Group, which Zara is the flagship event - and who wanted to be a business model which 'every company has something to learn'. Those studies, among other things, break up the logistics operation is becoming clear that the key to the success of this great chain of fashion. In contrast, in those explanations, workers are invisible. What working conditions employees have to bear the logistics? Is there an intensification of work because of the rhythms imposed? What role do unions play? Through this research has attempted to respond to these and other questions.

KEYwORDs: Logistic, flow of information, flexibility, working conditions, just in time, Zara.

\section{SUMARIO}

1. Una primera aproximación a Zara. 2. Logística: la clave del éxito en Zara. 3. El impacto del trabajo sobre los empleados de la logística. 4. Concluyendo.

\section{SUMMARY}

1. A first approach to Zara. 2. Logistics: Zara's success key. 3. Labour impact in logistics workers.

4. Concluding. 


\section{UNA PRIMERA APROXIMACIÓN A ZARA}

El Grupo Inditex (Industria de Diseño Textil, S.A.) hunde sus raíces en Confecciones GOA, S.A., la primera fábrica de confección de Inditex que fue creada en 1972. El negocio, dedicado a fabricar prendas de vestir (batas), fue creciendo hasta que, en 1975, apareciera Zara España, S.A., la primera sociedad de distribución y venta al detalle que tendría su primera tienda en la calle Juan Flórez, en La Coruña. ${ }^{2}$ Durante los años siguientes el negocio se expandió por las principales ciudades de España debido al éxito desatado entre su población -preeminentemente femenina-. ${ }^{3}$

Fue en 1985 cuando Inditex, S.A. (Industria de Diseño Textil, S.A.) se creó como eje del Grupo de empresas. A partir de entonces, centró sus esfuerzos de la producción en la cadena Zara. En los años sucesivos se ideó y llevó a cabo la implantación del sistema logístico, hecho a medida y con proyección de futuro. Así, se había implantado, ya a mediados de los años ochenta, un grupo textil que aglutinaba producción, logística y venta, como las tres piezas del engranaje que haría que la empresa llegara a ser un ejemplo de gestión para otros negocios.

A partir de ese momento y en los años sucesivos, la creación, adquisición y expansión de otras cadenas comenzó, mientras que Zara no hizo más que crecer en número, tanto dentro de España como en Europa y resto de continentes.

De este modo, en la actualidad, Inditex es uno de los principales distribuidores de moda del mundo y cuenta con nueve formatos ${ }^{4}$ diferentes: Zara, Pull \& Bear, Massimo Dutti, Bershka, Stradivarius, Oysho, Zara Home, Kiddy's Class y Uterqüe. ${ }^{5}$ Según los informes del Grupo, la 'creatividad y diseño esmerado, innovación, respuesta ágil al mercado, atención especial a la ambientación de las tiendas y una dirección empresarial flexible' han sido las claves de su éxito y expansión. ${ }^{6}$

CuAdro 1: Proceso de Producción de ZARA

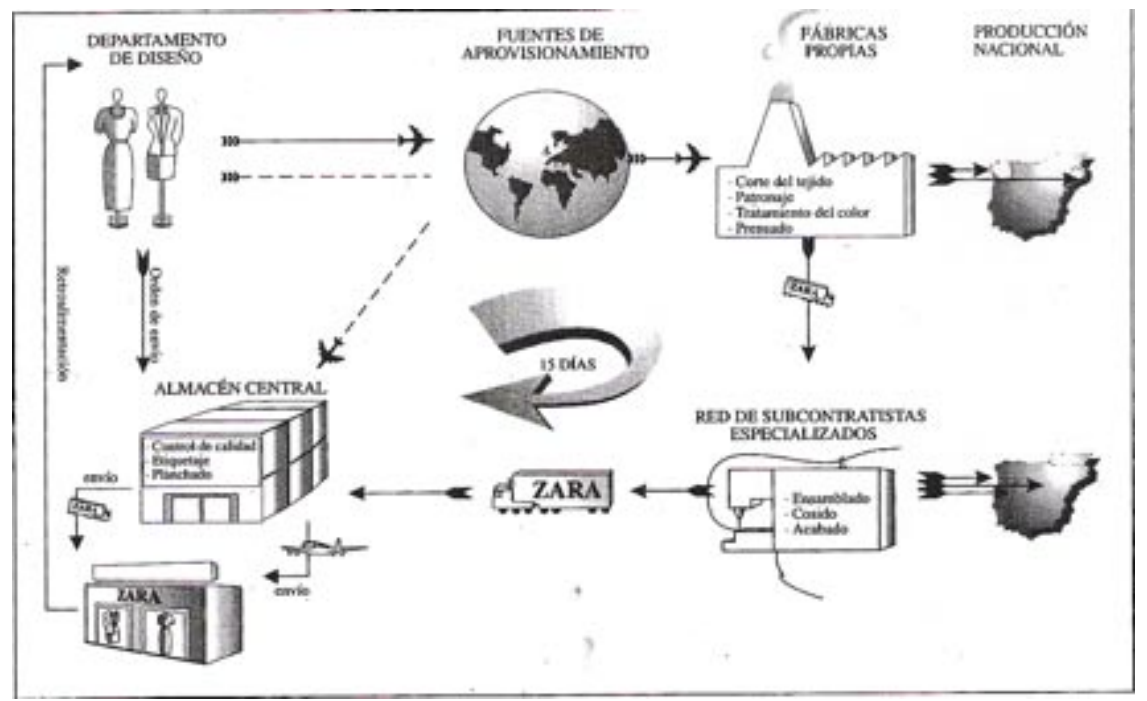

Fuente: Bonache Y CERVIÑO: 1996, 64

\footnotetext{
${ }^{2}$ Todavía hoy la primera tienda Zara sigue existiendo. A diferencia de la mayoría de las tiendas abiertas posteriormente, en ella siguen trabajando aquellas jóvenes que entraron por aquel entonces [entrevista realizada a Ana el 17-12-2007].

${ }^{3}$ [Informes Inditex, 1998-2010; Martínez Barreiro, 2008; Alonso Álvarez, 2000a; etc...].

${ }^{4}$ Es la forma con la que el Grupo Inditex se refiere a las diferentes marcas que ha ido creando y adquiriendo a lo largo de estos años.

${ }^{5}$ Junto a todos estos encontramos Lefties, que termina por vender todos aquellos productos que no han tenido cabida en el mercado normal, ni en el periodo de las rebajas. Su introducción en estas tiendas se realiza tras el cambio de etiquetas, modo a través del que se elimina el rastro de procedencia. Según la información que se ha podido obtener, las tiendas Lefties trabajan bajo el mismo número de CIF que Zara [Carmen Díaz: 12-03-2009].

6 [Informe anual 1998: 14, en www.inditex.es].
} 
Inditex comprende la mayor parte del proceso productivo: diseña, produce, distribuye y vende, tal y como se puede observar en el cuadro anterior.

El Grupo Inditex tuvo sus orígenes en un periodo en el que el discurso sobre la flexibilidad y reorganización del trabajo tomaba una gran importancia a nivel mundial. A finales de los setenta-principios de los ochenta, desde Europa, se abogaba por una nueva organización del trabajo en la que los recursos humanos adquirían una importancia clave a la hora de obtener mejores resultados empresariales. El estudio que aquí nos ocupa es una muestra de estos nuevos modelos productivos. ${ }^{7}$

A través de estos nuevos modelos productivos, mediante la participación de los trabajadores, de la rotación en diferentes puestos, se busca obtener empleados polivalentes capaces de llevar a cabo una mejora en los procesos de trabajo. Por ello resulta fundamental, entre otras cosas, la implicación y participación, puesto que desemboca en estrategias de calidad total. ${ }^{8}$ Lo que se trata con la introducción de los nuevos modelos productivos es de aligerar la organización y, de este modo, eliminar todo aquello que sea un excedente. ${ }^{9}$ Este será el método usado por Inditex en todas sus fases del proceso productivo. ${ }^{10}$

Teóricamente, calidad en el trabajo y el empleo suponen mejoras en la calidad de las condiciones del mismo.

Para comprobar si con la introducción de la nueva organización del trabajo, todos estos elementos se cumplen es necesario bajar al terreno y ver las condiciones reales. ¿Es cierto que se dan condiciones mejores que las habidas con el taylorismo?, o por el contrario, ¿al estudiar la realidad, se comprueba que hay intensificación del trabajo, descualificación, escasa autonomía de los trabajadores y de conocimientos formales (actitudes), etc., (elementos, todos ellos, que muestran la degradación de las condiciones de empleo y la precarización del mismo)?. Ello se puede ver en la serie de estudios realizados en el ámbito de la producción de las prendas de vestir, ${ }^{11}$ pero, ¿qué es lo que sucede en la logística?

La introducción de la producción ligera, del Justo a Tiempo (JAT), inserta en todos los procesos productivos del Grupo Inditex, buscará desde el punto de vista teórico la eliminación del despilfarro y la obtención de ganancias. Pero se ha de tener en cuenta que "(...) ninguna empresa es solamente un elemento de un sistema económico y social más vasto; cada empresa es, a su vez, un microsistema económico y social en el que muchos sujetos individuales y colectivos, con intereses y objetivos diferentes, entran en interacción.

Estos significa que cada empresa actúa en condiciones de equilibrio dinámico y precario, porque el cambio en las variables del contexto, exige ajustes continuos por parte de la empresa y porque, en el interior de la empresa, surge un proceso dialéctico entre intereses y objetivos diferentes, lo que exige negociaciones y mediaciones". ${ }^{2}$

El 'aligeramiento' de la producción supondrá el adelgazamiento de las plantillas y no la descarga de trabajo de la que se hablaba antaño. En palabras de Castillo, “(...) aligerar la producción puede ser aligerar el trabajo, esto es, meterle prisa, con los estímulos más colectivos que individuales, sobrecargarlo. Lo que técnicamente se llama "intensificación del trabajo". ${ }^{3}$ Veamos, pues, qué es lo que sucede en el caso que nos ocupa.

\section{LOGÍSTICA: LA CLAVE DEL ÉXITO EN ZARA}

'La creación de un centro logístico informatizado, que comunica la sede central del holding con cada uno de sus puntos de venta en el mundo

\footnotetext{
${ }^{7}$ Desde este caso de estudio se han podido analizar yestudiar las diferentes divisiones del trabajo, condicionadas por el tipo de producto realizado, en sus distintos niveles.

${ }^{8}$ [Castillo, 1996a , 1996b; Charro y Freyssenet, 1996; Cattero, 1996; Boyer y Freyssenet, 1996; Lahera Sánchez, 2001, 2004, $2005^{\mathrm{a}}$ y 2005 b; etc.].

${ }^{9}$ [Cattero, 1996].

${ }^{10}$ La procedimentalización del proceso productivo y la polivalencia de los empleados termina por hacer al trabajador más flexible y por tanto, prescindible debido a que todos terminan por ser intercambiables y ninguno resulta ser insustituible [Lahera, 2004].

${ }_{11}^{11}$ [Morales, J. y Villarino, M., 2007; Taboadela (et al.), 2005; Intermon Oxfam, 2004b].

12 [Volpanto (et al.), 1996: 136].

${ }^{13}$ [Castillo, 1996: 14].
} 
(...) flexibiliza la producción en la medida en que posibilita reponer el producto consumido-tallas, colores, patrones-, introducir en fábrica las modificaciones que dicta cada mercado especifico y conocer, además, en tiempo real la facturación de cada uno de esos puntos ' [Alonso Álvarez:, 2000b: 179].

Inditex pretende que las prendas se hagan a precios bajos, además de obtener la máxima rapidez en la entrega del producto que, una vez que se termina de fabricar y llega a Arteixo, recibirá los últimos retoques (planchado, etiquetado y embolsado) y será desde allí desde donde se distribuyan a todo el mundo. Lo que se busca es suministrar las prendas Justo a Tiempo haciendo competitiva a la empresa, al ser capaz de entregar la cantidad y variedad exactas en el mercado deseado.

La importancia de la logística, -amén del diseño, la fabricación y la venta- es otro de los elementos, que hace factible el modelo de negocio que Zara ha creado pudiendo asegurar que será el punto fundamental del modelo de éxito de la empresa. La entrega en plazos de tiempo muy breves, hace posible el que las prendas de las tiendas, se renueven con frecuencia. La reposición de las mismas se da dos veces por semana, no siendo superiores a setenta y dos horas los plazos de entrega de la mercancía. Ya sea por barco, autopista o avión, el producto sale al destino solicitado por la empresa y en el horario requerido por la misma. Las diferentes plataformas logísticas, -con su sede principal en Arteixo, -en el Polígono Sabón- y las de Zaragoza-, realizan un trabajo en que se busca ser eficientes -nuevamente- siendo capaces de acoplarse a los ritmos y volúmenes impuestos por los clientes. ${ }^{14}$ Así, del total del transporte, un $80 \%$ será terrestre, fórmula más eficiente para llegar en tiempo y costes, a los pun- tos de venta europeos. El transporte aéreo parece ser posible gracias a los acuerdos suscritos entre el Grupo y diversas compañías aéreas que hacen posible el que los tempos entre los pedidos y plazos de entrega se cumplan, no superando nunca las 72 horas. La posibilidad de contar con este tipo de transporte, hace viable el uso de los aviones para transportar la mercancía a los puntos más lejanos del planeta, haciendo que en el retorno carguen con suministros necesarios para la fabricación. El barco, en cambio, queda casi en exclusiva para el aprovisionamiento de materias primas, haciendo que los costes, por los plazos de entrega más dilatados, sean menores ${ }^{15}$.

Ya sea en un medio de transporte u otro, cabría mencionar la subcontrata en los mismos. Por un lado, en lo que se refiere al transporte por carreteras, la empresa Azkar será la encargada de realizar los portes. En cuanto a los aviones, la empresa ha llegado a diferentes acuerdos con diferentes aerolíneas (KLM-Air France) que serán las encargadas de llevar la mercancía a los destinos solicitados ${ }^{16}$. Aún así, en el año 2006 aparecía una noticia en la prensa nacional en la que se decía, que entre otras de las estrategias del Grupo en su plan de ajuste, Inditex tenía intención de reducir sus gastos al máximo en lo que a transporte y logística subcontratada se refiere, con la intención de alcanzar mayor eficiencia en sus costes. ${ }^{17}$

Pero a pesar de la importancia del transporte, el sistema logístico del grupo no empieza ni acaba en éste, va mucho más allá... La logística, siendo la distribución física de los productos terminados con el consiguiente reparto final a las tiendas, ${ }^{18}$ también será ' $f u$ jos de información que se ponen en marcha, ${ }^{19}$ con el fin de dar al consumidor el nivel de servicio adecuado a un coste razonable'.$^{20}$ Por tanto, cabría preguntarse dónde empieza y dónde termina el sistema logístico de Inditex. La central del Grupo mantiene la conexión, en

\footnotetext{
${ }^{14}$ El Grupo Inditex cuenta con otros puntos logísticos por la geografía española, pero estos surten al resto de marcas. Así, los dos centros logísticos que se ocupan de surtir a las tiendas Zara de todo el mundo, se encuentran en Arteixo, en el polígono Sabón, donde nació Zara, y en Zaragoza, en la Plataforma logística de PLAZA.

${ }^{15}$ [Badía, 2008].

16 [Bonache y Cerviño, 1996; Alonso Álvarez, 2000b].

17 [www.elmundo.es, visto el 19 de septiembre de 2007].

${ }^{18}$ [Alas-Pumariño y Fernández, 2005].

${ }^{19}$ La negrita es nuestra.

${ }^{20}$ Ballou, Ronald H. (1999) Business Logistics Management, Cuarta edición, Upper Saddle River (Nueva Jersey, EE.UU.): Prentice-Hall International, Inc.. ISBN 0-13-081262-5. en http://es.wikipedia.org/wiki/Log\%C3\%ADstica\#cite_note-1 [visto el 21 de octubre de 2009].
} 
tiempo real, con todas las tiendas del mundo. Desde los almacenes a las tiendas, a través de sistemas informáticos que se han ido adaptando al momento.

Zara parece ser la primera empresa que ha integrado un sistema que se basa en Internet a través del que se dan flujos de información entre las tiendas, la sede central y los talleres y cooperativas que trabajan para el Grupo, tal y como aparece en el Cuadro 2. De esta manera la comunicación es continua y permite el que se procese toda la información: desde el departamento de diseño a las fábricas, pasando por los suministros que han de llegar a éstas, que harán posible el que las nuevas prendas estén en las tiendas en el plazo de dos semanas.

Cuadro 2: Modelo ZARA

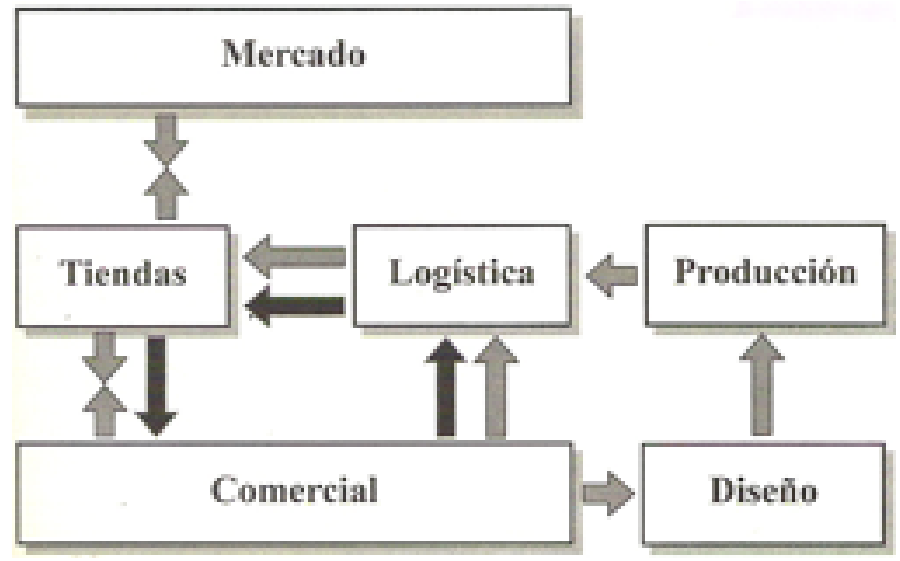

FuENTE: BADÍA 2008, P. 121

En los primeros años del Grupo, relata Badía, la información se trasladaba a través de faxes, hasta que la expansión fue tal que el sistema se colapsaba. Por ello se optó por un tipo de PDA, la Newton, mediante la que los encargados de las tiendas podían y pueden realizar los pedidos, recibir diferente información sobre lo que va a llegar a las tiendas, etc... Pero, para un mejor funcionamiento del sistema, en el año 2006, el Grupo empresarial incorporó los Terminales de Gestión de Tiendas (TGT). El TGT está dotado de un programa informático que hace posible que el personal de las tiendas, a través de una pantalla táctil, pueda tener acceso a todo tipo de información sobre los productos, sobre el almacén, mantenerse comunicados con la dirección de cada cadena, con los centros logísticos y con otras tiendas, mediante el correo electrónico. De este modo, la relación con el cliente se vuelve mucho más ágil.
Además de ello, parece ser evidente la mejora que se puede dar en la gestión comercial puesto que el TGT, que se va implantando poco a poco en todo el mundo, posibilita el realizar programas on-line, para los empleados en tienda, de formación. ${ }^{21}$

Los datos de caja-ventas- de todas y cada una de las tiendas llegan diariamente a la sede central de cada cadena, transmitidos nada más concluir el horario de apertura al público... [Badía, 2008:118].

A través de la PDA, o Casiopea, las dependientas tienen la posibilidad de mantener un contacto continuo con la central de A Coruña en tiempo real. Desde ella se hacen los pedidos de lo que se quiere que haya en las tiendas, manteniendo el contacto mediante correos electrónicos. De este modo la central de Zara conoce en todo momento aquello que sucede en las

${ }^{21}$ [Informe 2006-2007]. 
tiendas de todo el mundo: desde las prendas que se venden y devuelven hasta el stock existente en los almacenes de cada uno de sus negocios.

Por tanto, se podría decir que la logística parte desde las mismas tiendas, en el momento en que se cursan los pedidos y con el desarrollo de plazos que van de las 24 a las 48 horas entre la solicitud y la entrega, siendo, hasta de setenta y dos horas en los lugares más remotos. Inmediatez y rotación de prendas parecen ser dos de los elementos cruciales para la eficiencia de Zara. Por tanto se dará una distribución de circuito corto, en que se surtirá a las tiendas con mayor frecuencia (dos veces por semana) pero en cantidades menores. La política seguida por Zara es la de satisfacer las tendencias de los consumidores, elemento conseguido a través de la información que la central recibe, a diario, desde las tiendas. Una vez que termina el día, mediante el arqueo de caja, se manda a la central las ventas realizadas -prendas, colores, tallas y precios concretos- de lo que se deduce el stock restante en el almacén..$^{22}$ De este modo se conocen las preferencias de las diferentes zonas pudiendo intercambiar determinados productos entre establecimientos para conseguir el stock cero en todos los negocios. Además de este tipo de información, según lo dicho en los informes del Grupo y diversos artículos, los datos se complementan con los comentarios que los propios empleados hacen llegar a la central sobre los gustos y preferencias de los clientes atendidos. Revirtiendo en una rápida reposición de las tiendas, que hará posible el que se reduzcan los costes financieros que normalmente derivan del almacenaje y consecutivo mantenimiento. Gracias a que es posible, de este modo, adaptar los productos a la demanda de los consumidores, así como adecuarla: desde las tallas, a los colores que más se solicitan. ${ }^{23}$

En términos generales (...) la central remite a las tiendas un catálogo de prendas al inicio de cada una de las tres temporadas, que acaba suponiendo en torno al $60 \%$ de las existencias que integrarán la oferta del periodo. Será a partir de ahí que los encargados irán confeccionando los pedidos bisemanales, planteando retiradas, ampliaciones y sustituciones de unas prendas $u$ otras, por lo general aplicando el principio de devolver a la central toda prenda que permanezca sin venderse por espacio de dos semanas [Badía, 2008: 274].

La lejanía del polígono Sabón, desde el que se distribuían las prendas a las tiendas de toda España, no parecía resultar una dificultad para hacer llegar los modelos en los plazos previstos por el Grupo. Pero en 1995 se informatizó totalmente el centro logístico y se creo un sistema de telecomunicaciones integrado, para mantener en comunicación la central con los centros de aprovisionamiento, producción y venta de todo el mundo. ${ }^{24}$ Aún así, la expansión de Zara por Europa y resto del mundo -y por tanto la necesidad de mayor número de metros cuadradoshizo que la empresa tuviera que adquirir nuevos espacios. En el año 2000, en el mismo polígono en que había iniciado sus andaduras, Inditex instaló sus servicios centrales. Desde este centro logístico, en el año 2000, se distribuían 60.000 prendas plegadas a la hora, junto a la ropa que iba por los carriles aéreos hasta los muelles de carga. Desde dicho centro, -mediante el transporte terrestre, que recorre más de 6,7 millones de kilómetros, y el aéreo, que carga una media de 7,8 millones de kilos de prendas-, salían 1,8 millones de prendas a las tiendas Zara de todo el mundo. ${ }^{25}$

El centro logístico de Arteixo ha sido y sigue siendo la referencia: funciona de forma satisfactoria y acumula años de ajuste y optimización. Tras sucesivas ampliaciones, rebasa los 400.000 metros cuadrados de superficie y ocupa alrededor de 1.000 personas, de las 3.500 que integra la plantilla total de Inditex en el Polígono Sabón, y está conectado con las dieciocho plantas que surten la ropa de Zara a través de varios túneles $y$ en torno a $250 \mathrm{~km}$. de carriles automatizados [Badía,2008: 170].

\footnotetext{
${ }^{22}$ En este punto cabría recordar que cada prenda lleva un código de barras que 'permite seguir el estado de un pedido en cualquiera de sus fases obteniendo la información en tiempo real' [Martínez Barreiro, A., 2008: 112].

${ }^{23}$ [Alonso Álvarez, 2000b; Bonache y Cerviño, 1996; Castellano, 2002].

${ }^{24}$ [Alonso Álvarez, 2000].

${ }^{25}$ El País Negocios (2000) Un particular estilo de vida, domingo 29 de octubre de 2000. Edición impresa.
} 
A pesar de su política de cercanía entre las sedes y el centro de distribución, tres años más tarde, en 2003, se abría el segundo centro de distribución de Zara, en PLAZA (Plataforma Logística de Zaragoza), en Zaragoza a pocos kilómetros de la ciudad. Éste resulta tener una posición estratégica dentro de la geografía española: próximo a la ciudad de Zaragoza, cuenta con la cercanía a la red de carreteras, al aeropuerto y trenes. Mediante este nuevo centro se complementaba la actividad que ya venía ejerciendo el centro logístico de Arteixo, siendo éste, el nuevo distribuidor para Europa, Oriente Medio y Asia. Dicho centro ocupa una superficie de 125.000 metros cuadrados. Pero en 2005 se abrieron otros dos nuevos, estos, situados en León ${ }^{26}$ y Meco que venían a sumarse a los anteriores. ${ }^{27} \mathrm{El}$ primero para poder hacer frente a las prendas de moda en proceso de liquidación, el segundo para las prendas infantiles y las del hogar.

El sistema logístico, por tanto, consigue ser el nexo de unión entre los diferentes procesos de diseño, compra, producción, suministro y rotación de prendas, a través de los canales de información que se mantienen abiertos. Por tanto, la logística es información fluida, es procesamiento, es almacenaje, es transporte... es la pieza fundamental del engranaje. Aquella capaz de haber reducido el tiempo de acceso de las prendas al mercado en más del $80 \% .^{28}$ Por tanto la logística sería capaz de paralizar todo el conglomerado que da vida a Zara.

Según el Grupo:

El modelo de negocio de Inditex se caracteriza por la búsqueda de la flexibilidad en la adaptación de la oferta comercial a la demanda del mercado, mediante el control de la cadena de suministros en sus distintas fases de diseño, fabricación y distribución, lo que proporciona la capacidad de enfocar la producción propia o de proveedores a los cambios de tendencia dentro de cada campaña comercial' [Informe 2006:79].

\section{EL IMPACTO DEL TRABAJO SOBRE LOS EMPLEADOS DE LA LOGÍSTICA}

"En el centro logístico (el de Sabón), comunicado por túneles con sus fábricas, hay un gigantesco mecano automatizado $-210 \mathrm{~km}$ de troles- por el que viajan las prendas colgadas o dobladas, que a través de lectores ópticos se almacenan por talla, color y destino (país y tienda). Esta maquinaria, diseñada por la empresa y fabricada por Toyota, trabaja permanentemente $y$ distribuye 60.000 prendas/hora. Desde el centro logístico envía la mercancía, a través de servicios subcontratados de transporte a los que se les entrega los trámites aduanales ya realizados, a las tiendas de España, América y Asia, y desde el de Zaragoza al resto de Europa" [Morales y Villarino, 2007: 12-13].

Como se ha podido comprobar la información y el tiempo de respuesta, desde que se diseña la prenda hasta que aparece en la tienda, resultan ser dos de los elementos cruciales en el modelo creado por Zara-Inditex.

La logística es una de las partes más importantes en la consolidación y crecimiento del Grupo Inditex:

"Lo importante es la logística" [Montserrat: 18-12-2008, 8].

Entre otras cosas se resalta la importancia de un sistema centralizado, mediante el cual se hacen los pedidos a la vez que se atiende al cliente. De esta manera, desde los almacenes de logística saben qué se tiene que reponer en cada lugar, si compensa o no reenviar algunas de las prendas o si interesa hacer un movimiento de los productos entre tiendas. Por tanto, a la vez que venden se va haciendo el pedido a la fábrica.

Una vez que la prenda está terminada, -planchada, con los últimos retoques hechos y embolsada-, se ha de cargar en los muelles del centro logístico

\footnotetext{
${ }^{26}$ Especializado en productos con marca Lefties.

${ }^{27}$ Todos los centros logísticos, aquellos situados en Cataluña, Galicia y Meco, exceptuando los de Arteixo y el de PLAZA, se dedican a la distribución de prendas de los demás formatos pertenecientes al Grupo.

${ }^{28}$ Zara diseña y fabrica en 3-4 semanas -frente a la competencia que necesita alrededor de 6 meses para el diseño y 3 meses para la fabricación y puesta en tiendas - y lo distribuye en siete días [Martínez Barreiro, 2008].
} 
para enviarla a los destinos requeridos. Las prendas se mueven en carruseles, a través de cintas automatizadas, cayendo en las cajas previstas, previo paso de los lectores que las seleccionan. El orden de las cajas aparentemente es aleatorio pero los carruseles, que llevan un letrero con el destino al que deben ir los modelos que éste contiene, terminarán por corresponderse con la caja que lleva el mismo cartel. De vez en cuando, como las cajas van sobre una cinta, se mueven para que la mercancía que entra se coloque y así evitar que toda las ropa termine agolpada en un lado de la caja. En lo que respecta a la prenda colgada, más o menos se sigue el mismo proceso. Ya sean cajas, ya sean prendas colgadas, todo va directamente al camión que se dirige a la tienda correspondiente. Una vez llegado al negocio, el vehículo se descarga en el almacén, dejándose las cajas y los 'burros' dispuestos para que las dependientas de la tienda, se dispongan a sacar la ropa y colocarla, antes de la apertura de la misma. Este proceso se da dos veces por semana. Podría decirse que existe una continuidad clara entre todos los procesos de Zara-Inditex: diseño, producción, distribución y venta. Todo está interconectado.

La rapidez resulta esencial en un modelo que se basa en el JAT y ello debe afectar a los ritmos de trabajo de los empleados del Grupo, haciendo que sus condiciones reales estén marcadas por la precariedad y la intensificación.

El proceso que se ha narrado anteriormente, conlleva una serie de condiciones laborales que los propios afectados vive a diario. Si bien es cierto que el centro logístico está compuesto por zonas completamente automatizadas, también lo es el que las mismas generan un trabajo más intensificado entre los empleados, debido a que deben seguir los ritmos que éstas marcan.

"Las máquinas que hay, se llaman carrusel y son de reparto, son para hacer el trabajo mucho más rápido y eliminar puestos de trabajo" [Juan Díaz: 21-01-2008].

Las máquinas que reparten la paquetería, la confección y las prendas colgadas, pueden repartir entre 25.000 y 30.000 prendas a la hora. Se trata de una maquinaria de grandes dimensiones que contiene una serie de recipientes que los trabajadores llaman tolvas. En estos departamentos van las cajas, que corresponden cada una a una tienda. Cada caja se abre y gracias al funcionamiento de unas paletas que giran, la ropa cae. El operario debe ir colocando las cajas y empujarlas una vez que éstas están llenas, poniendo una nueva caja para que las prendas sigan cayendo. Las que terminan de llenarse continúan el recorrido a través de una cinta, hasta llegar a una precintadora que se encarga de cerrarlas mediante un precinto en el que hay una pegatina que identifica el destino al que se dirigen. Posteriormente existen unos desvíos por los que caen. Así, el operario, va colocando la mercancía identificada en el carro correspondiente a la tienda a la que se ha de mandar. Una vez que los carros están completos se traslada a los camiones, donde la mercancía se carga a mano.

Por todo ello, los propios trabajadores se quejan de una falta de inversión en determinados procesos, si bien reconocen que en otros, sí se está gastando en tecnología.

"Sí están invirtiendo en infraestructura pero no todo lo que deberían. Se siguen cargando los camiones a mano" [Juan Díaz: 21-01-2008].

El sistema de logística de Inditex, el de Zara, según sus trabajadores, es un sistema de "reposición seguida", esto es, de forma diaria se reponen las prendas que desde las tiendas se solicitan:

"Tú pides una prenda hoy y mañana la tienes en tienda, porque te la sirven" [Juan Díaz: 2101-2008].

Por otra parte, como se ha explicado anteriormente, mediante el Terminal de Gestión de Tiendas (TGT), las encargadas de los diferentes establecimientos realizan la demanda de productos al departamento comercial. Los encargos llegan directamente a un ordenador y desde allí, desde Comercial, se hace llegar la cantidad requerida al almacén desde el que se "sacan los pedidos" y se reparten por las tiendas de todo el mundo, sin superar la entrega el límite de dos días. ${ }^{29}$

\footnotetext{
${ }^{29}$ Exceptuando aquellos modelos que, en determinadas ocasiones, están agotados debido a que son series limitadas que una vez que se terminan no se vuelven a producir.
} 
Pero dicho método de trabajo perjudica a los propios empleados de logística debido al exceso de horas extraordinarias que se ven obligados a realizar para conseguir que el sistema de JAT funcione sin ningún tipo de fisura. El número de prendas, generalmente, supera la cantidad que los propios trabajadores saben que pueden llegar a cumplir en una jornada de trabajo normal. Por lo que si no consigue sacarlo adelante en dicho plazo, los empleados tendrán que alargar su jornada, el tiempo que sea necesario.

Además, todavía hoy los camiones se cargan a mano. Desde el almacén, las prendas se organizan en palés y se van metiendo manualmente en los camiones. Es el mozo el que descarga las cajas desde el almacén al camión, organizándolo por colores y tallas. En cambio, otros vehículos se cargan mediante planchas. Éstas se meten en el camión para posteriormente descargarlas en los aviones que llevarán las prendas a los destinos en los que se hizo el pedido.

La flota de vehículos que el Grupo usa para los portes que ha de realizar por España y resto de Europa pertenece a una subcontrata, ${ }^{30}$ al igual que la de descarga de los vehículos en las tiendas de destino. ${ }^{31}$ EUROCEN ha sido la encargada de bajar la mercancía de los camiones, quitar los plásticos y dejar la ropa "libre" para que las dependientas de las tiendas, terminaran de colocar lo recibido antes de la apertura de las tiendas.

En 2008, Zara, había comenzado a probar un nuevo método en una serie de tiendas usando a personal de la plantilla. La intención era la de emplear a trabajadores contratados por la propia empresa para realizar el trabajo que hasta entonces venía haciendo la subcontrata anteriormente mencionada. Así, en enero de 2008, se estaban realizando pruebas en diecisiete tiendas de toda España, entre las que había una tienda en Ferrol, la del Centro Comercial Odeón, otra en San Sebastián, otra en Asturias, otra en Málaga,... y así, hasta llegar a las diecisiete. Estas pruebas piloto tenía el fin de comprobar si dicho sistema funcionaba y en caso afirmativo, ir instalándolo, poco a poco, en todas las cadenas del Grupo Inditex. Con la implan- tación de este nuevo método la empresa pretendería evitar el trabajo que las dependientas tienen que realizar dos veces por semana y antes de la apertura de las tiendas, una vez que los camiones han descargado. Mediante este sistema las tiendas tendrían la mercancía colocada, de tal forma que todas las empleadas estuvieran en el área de venta. Los nuevos trabajadores tendrían una jornada de dos de la madrugada a diez de la mañana, pudiendo así descargar y colocar, evitando que los días de reposición entre cuatro y cinco personas permanezcan en el almacén, colocando en la tienda como aún hoy viene sucediendo. Con este nuevo sistema sólo habría gente en el almacén para la reposición de los artículos.

La cantidad de trabajadores en los almacenes de logística del Polígono de Sabón puede llegar a las mil personas. La inmensa mayoría, todavía hoy, son hombres y aunque en la actualidad, a diferencia de las fábricas y de las tiendas, no hay tantas mujeres trabajadoras, el número de éstas se ha incrementado, siendo de en torno a un $40 \%$ de la plantilla según uno de los entrevistados. ${ }^{32}$ Según datos del año 2006, el personal de logística en Arteixo estaba integrado por alrededor de 900 trabajadores habiéndose, en ese mismo año, incrementado el número de indefinidos en 250. Esto hacía que los empleados de esta área estuviesen más tranquilos debido a que la temporalidad parecía que comenzaba a ser utilizada en los casos en que hubiese necesidades puntuales, usando la indefinida para las necesidades estables. ${ }^{33}$ Pero, en el mismo año, los empleados mostraban su descontento debido a una 'discriminación retributiva' del convenio e instaban a un acto de protesta. ${ }^{34}$

Dentro de la logística, parece ser que entre los trabajadores de almacén existe una conciencia sindical más fuerte que las que hay en otras áreas de la empresa. Una de las razones que podrían explicar este hecho, es que los de la logística, no encontrándose dispersos por diferentes espacios, como les sucede a las dependientas de las tiendas, son capaces de organizarse mejor, de conocer los problemas de todos los compañeros, de realizar acciones conjuntas. De este

\footnotetext{
${ }^{30}$ Montserrat entrevista realizada el 18 del 12 de 2008.

${ }^{31}$ Entrevista a Juan Díaz el 21 de enero de 2008.

${ }^{32}$ Entrevista a Juan Díaz el 21 de enero de 2008.

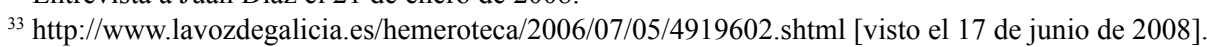

${ }^{34} \mathrm{http}$ ://www.abc.es/hemeroteca/historico-13-05-2006/abc/Galicia/los-trabajadores-de-zara-logistica-denuncian-la-discriminacionretributiva-del-ultimo-convenio_1421537944576.html [visto el 27-08-2007].
} 
modo, pueden construir una conciencia colectiva a través de la que tratan de enfrentarse a los incumplimientos empresariales en materia laboral y llegar a negociar determinados asuntos con la empresa.

Así, las condiciones de éstos parecen ser algo mejores que las de las vendedoras y de los de la fabricación, puesto que van obteniendo mejoras a través de la negociación. En 2007 consiguieron firmar un acuerdo por el que se obtuvo una subida salarial de casi un $12 \%$ a la vez que la reducción de la jornada para mayores. Por ello, los representantes de los trabajadores y los empleados parecían estar satisfechos:

"En almacén podemos estar medianamente más contentos. Ahora firmamos un acuerdo, con subida salarial bastante considerable, casi un $12 \%$ además de una reducción de jornada a mayores. Y bueno, pues sí, está bien." [Juan Díaz: 21-01-2008].

Pero a pesar de que entre estos trabajadores hay una actividad sindical fuerte, a medida que se van construyendo nuevas plataformas la sensación de unión se va diluyendo:

"Antes estaba todo concentrado aquí (en Arteixo), lo que suponía un poder de movilización sindical mucho mayor, y ahora, estamos hablando de cuatro plataformas de Zara en toda España y eso, para movilizarnos, es mucho más complicado. Porque pueden desviar el trabajo de uno para otro. La central sigue siendo esta, el que más factura sigue siendo este y el más grande... Pero con el tiempo, eso se va a acabar" [Juan Díaz: 21-01-2008, 8].

\section{CONCLUYENDO}

Mediante este texto se ha querido hacer llegar al lector la importancia que ha adquirido la logística en el modelo de Zara-Inditex. Hemos podido demostrar que aquella ha pasado a ser la pieza fundamental para el perfecto funcionamiento del proceso completo de producción que engloba el Grupo.

A medida que se ha ido avanzando en la investigación hemos llegado a ser conscientes de que la logística es el elemento que ha llevado a Zara a ser el modelo empresarial admirado y tratado de imitar por otras grandes cadenas de moda. Logística ya no es sólo el transporte y almacenaje sino que también lo es la 'información fluida' que se da entre los diversos procesos productivos: puntos de venta, diseño y fabricación. Con este sistema de información, mediante el que todo está interconectado, se consigue un 'aligeramiento' de cualquiera de los procesos.

1- De un lado, la cantidad de producto, en los almacenes de Sabón y PLAZA y en las tiendas, es menor que antaño. Ello, como hemos podido comprobar, se consigue mediante la distribución de circuito corto, mediante el sistema de reposición seguida. Esto es, se reponen menos cantidades de prendas pero, dos veces por semana y conforme a los pedidos realizados desde las propias tiendas. Así se consigue un stock cero, esto es: todo lo que hay en las tiendas se vende y no hay excedente, obteniendo mayores ganancias y eliminando el despilfarro.

En contraposición, comprobamos que los empleados de la logística han de realizar horas extraordinarias para poder llegar a cumplir con los pedidos realizados desde las tiendas, debido a que el número de prendas solicitado suele superar al número de horas de una jornada normal.

2- Hemos podido comprobar cómo desde la empresa se habla de la informatización de los centros logísticos, elemento que hará posible un aligeramiento de este proceso.

En cambio, descubrimos que no todo está informatizado y que el hecho de que determinadas partes sí lo estén, ha hecho posible una reducción o adelgazamiento de la plantilla, elemento que conlleva una intensificación del trabajo de los empleados, que ven cómo sus condiciones se precarizan: han de trabajar más horas, realizar, en muchas ocasiones, lo suyo y lo de sus compañeros que ya no están en la empresa y seguir los ritmos marcados por las máquinas para llenar a mano las cajas y los camiones.

3- Hemos entendido la logística como el eje para que el 'modelo Zara' siga siendo un éxito. Para ello, la rapidez será esencial pero, como hemos podido corroborar, rapidez y aligeramiento suelen llevar implícito un significado negativo. Los trabajadores sufren condiciones de trabajo precarias e intensas.

Por este motivo los sindicatos reivindican una serie de mejoras: en los sueldos, en las jornadas, etc... Y el hecho de una mayor unidad sindical en los 
centros logísticos -debido a que no se encuentran tan dispersos como los trabajadores de las tiendashace más factible el llegar a obtener la mejora de las condiciones laborales.
En definitiva el análisis documental ha dado muestra de la intensificación laboral, de la precarización de las condiciones de trabajo y de todo lo que ello conlleva en el ámbito de la logística.

\section{BIBLIOGRAFÍA}

AA. VV. (2010) La investigación y la enseñanza de la sociología del trabajo. Un balance de la situación en España, Valencia, Germanía [ISBN: 978-84-92587-38-4].

Alas-Pumariño, A. y Fernández Gómez, J. (2005) "Externalización de procesos y trabajo autónomo: los transportistas de mercancías en el sector de logística y distribución" en Castillo, J.J. (2005) El trabajo recobrado. Una evaluación del trabajo realmente existente en España, Madrid-Buenos Aires, Miño y Dávila, pp.79-122.

Alonso Álvarez, L. (2000a) "Inditex-Zara. Vistiendo a tres continentes" en Ojeda, F. (coord.), Grandes empresas, grandes historias de Galicia, A Coruña, Voz de Galicia.

Alonso Álvarez, L. (2000b) "Vistiendo a tres continentes: la ventaja competitiva del grupo Inditex-Zara 1963-1999", en Revista de Historia Industrial, $\mathrm{n}^{\circ}$ 18, pp.157-179.

BADíA, E. (2008) Zara ... y sus hermanas, Madrid, LID.

Ballou, Ronald H. (1999) Business Logistics Management, Cuarta edición, Upper Saddle River (Nueva Jersey, EE.UU.): Prentice-Hall International, Inc.. ISBN 0-13-081262-5. en http://es.wikipedia.org/wiki/Log\%C3\%ADstica\#cite_ note-1.

Bellusi, F. (1992) “La flessibilità si fa gerarchica: la Benetton” en Bellusi, F. (ed.) (1992) Nuovi modelli di Impresa, Gerarchie Organizzative e Imprese Rete, Milán, Franco Angeli.

Blanco, F y Redondo, P. (2000) "Inditex (1994-1999)" en Munuera Alemán, J.L. y Rodríguez Escudero, A.I. (2000) Estrategias de marketing para un crecimiento rentable. Casos prácticos, Madrid, ESIC Editores.

BoIx, I. (2007) "Los derechos del trabajo en las cadenas de producción de las empresas multinacionales" en Observatorio sociolaboral, 57, julio 2007 en http://issuu.com/fundacionse/docs/observatorio2007/57.

Boltanski y Chiapello (2002) El nuevo espiritu del capitalismo, Madrid, Akal.

Bonache, J. y Cerviño, J. (1996) "Caso Zara: el tejido Internacional”, en Durán Herrera, J.J. (coord.) (1996) Multinacionales Españolas I. Algunos casos relevantes, Madrid, Pirámide, pp. 51-58.

Boyer, R. y Freyssenet, M. (1996) "Emergencia de nuevos modelos industriales. Problemática del programa internacional de GERPISA", en Sociología del Trabajo, no 27, pp. 23-54.

Burawoy, M. (1985) El consentimiento en la producción. Los cambios del proceso productivo en el capitalismo monopolista, Madrid, Ministerio de Trabajo y Seguridad Social.

Castellano, J.M. (2002) "El proceso de internacionalización de Inditex", en Boletín económico ICE, n 799, abrilmayo, pp. 209-217.

Castellano, J.M. (1993) "Una ventaja competitiva: el factor tiempo. El caso Inditex-Zara" en Papeles de economía Española, ${ }^{\circ}$ 56, pp. 402-404.

Castellanos, M. L. y Pedreño, A. (2006) Los nuevos braceros del ocio: sonrisas, cuerpos flexibles e identidad de la empresa en el sector turístico, Madrid-Buenos Aires, Miño y Dávila.

CAstillo, J.J. (2005) El trabajo recobrado. Una evaluación del trabajo realmente existente en España, Madrid-Buenos Aires, Miño y Dávila.

Castillo, J.J. (1996a) "Presentación: "Un fantasma recorre Europa"... de nuevo, la producción ligera", en Sociología del Trabajo, $\mathrm{n}^{\circ} 27$, pp. 3-22.

CAstillo, J.J. (1996b) "Fabricando la organización del trabajo de mañana: una fábrica líder en la mecánica", en Sociología del Trabajo, $\mathrm{n}^{\circ} 27$, pp. 55-76.

CAttero, B. (1996) “¿Solamente despilfarro? Sobre redundancia y “slack” en la producción ligera”, en Sociología del Trabajo, no 27, pp. 77-102.

Charron, E. y Freyssenet (1996) "La "producción reflexiva" en la fábrica Volvo de Uddavalla", en Sociología del Trabajo, $\mathrm{n}^{\circ} 27$, pp. 103-130.

CoRIAT, B. (1993) El taller y el robot;: ensayos sobre el fordismo y la producción en masa en la era electrónica, Madrid, Siglo XXI. 
El Mundo (2006) Inditex ahorrará 300 millones con su plan de ajuste hasta 2008, martes 11 de abril de 2006 . En www. elmundo.es visto el 16 de septiembre de 2007.

FÁbrega, F. (2004) ZARA. El modelo de negocio Inditex, Madrid, Claves de Gestión.

Flavián Blanco, C. y Polo Redondo, Y. (2000) “Inditex (1994-1999)”, en Munuera Alemán, J.L. y Rodríguez Escudero, A.I. (2000) Estrategias de marketing para un crecimiento rentable. Casos prácticos, Madrid, Esic Editores.

GerefFi, G (2001) "Las cadenas productivas como marco analítico para la globalización”, en Problemas del desarrollo, México, IIEC-UNAM, Abril-junio. Vol. 32, n. 125, pp. 9-37.

Herreros de las Cuevas, C. (2005) Zara: un reto al pensamiento maduro en http:/www.gestiondelconocimineto.com/ documentos $2 /$ carlos_herreros/caso_zara.htm.

INDITEX, INFORMES ANUALES 1998-1999-2000-2001-2002-2003-2004-2005-2006-2007-2008 www.inditex.es.

Instituto Nacional de Estadística: www.ine.es.

InTERMON OXFAM (2004a) Más por menos. El trabajo precario de las mujeres de las cadenas de producción globalizadas, www.comercioconjusticia.com [visto el 13 de enero de 2006].

InTERMON OXfam (2004b) Moda que aprieta. La precariedad de las trabajadoras de la confección y la responsabilidad social de la empresa, www.IntermonOxfam.org [visto el 14 de marzo de 2007].

Jiménez, M. (1999) "Una logística condicionada por la moda” en Súper Aral Lineal, 1999, n. 1423, pp.40-42.

JiMÉNEZ, M. (1999) “Cuestión de moda” en Logística profesional, año 3, n 26, 1999, pp. 28-33.

LAHERA SÁNCHEZ (2005a) "Mutaciones productivas, trabajo y empleo: ¿desarrollando la cualificación y las competencias de los recursos humanos?", en Fundación Tripartita paras la Formación en el Empleo (2005) Competencias, igualdad de oportunidades y eficacia de la formación continua, Madrid, Fundación Tripartita, pp. 161-208

Lahera SÁnchez, A. (2005b) Enriquecer el factor humano: Paradigmas organizativos y trabajo en grupo, Barcelona, El Viejo Topo.

LAHERA SÁNCHEZ (2004) La participación de los trabajadores en la democracia industrial, Madrid, La Catarata.

LA VOZ DE GAlicia (2006) Inditex hace fijos a 250 empleados de Zara Logística, garantizando el futuro del centro de Arteixo, 5 de julio de 2006 en http://www.lavozdegalicia.es/hemeroteca/2006/07/05/4919602.shtml [visto el 17 de junio de 2008].

LóPEZ CAlle (2007) La desmovilización general. Jóvenes, sindicatos y reorganización productiva, Madrid, La Catarata.

Martínez Barreiro, A. (2008) "Hacia un nuevo sistema de la moda. El modelo Zara", en Revista Internacional de Sociología, vol. LXVI, no 51, pp. 105-122.

Martínez Barreiro, A. (2004) "Moda y Globalización: de la estética de clase al estilo subcultural”, en Revista Internacional de Sociología, $\mathrm{n}^{\mathrm{o}}$, pp.139-166.

Martínez Barreiro, A. (1998) Mirar y hacerse mirar. La moda en las sociedades modernas, Madrid, Tecnos

Molas, J. (1994) “Aplicaciones telemáticas en el sector textil-confección”, en Información Comercial Española, 726, pp.141-159.

Morales, J. y Villarino, M. (2007) “Moda y maquila. El trabajo precarizado en la globalización”, en Sociología del Trabajo, $\mathrm{n}^{\circ}$ 59, pp.7-34.

Pita Wonenburger, X. (2007) Inditex prevé que la batalla de los costes siga, en http://www.xabierpita.es/2007/03/ inditex_preve_que_batalla_de_1.php [visto el 15 de junio de 2007].

Taboadela, O.; CAStro, M. y MARTínez, M (2005) "Del escaparate a la trastienda: mujeres y trabajo invisible en la confección gallega" en Castillo, J.J. (2005) El trabajo recobrado. Una evaluación del trabajo realmente existente en España, Madrid-Buenos Aires, Miño y Dávila, pp.171-219.

VÁzquez García (2000) “El modelo Zara”, en González, L. et al. (2000) La industria de la moda en Galicia, Santiago, Instituto de Estudios e Desenvolvemento de Galicia [DEGA], pp.73-89. 